\title{
Treatment Experiences with CDK4\&6 Inhibitors Among Women with Metastatic Breast Cancer: A Qualitative Study
}

\author{
Judith J Stephenson $\mathbb{D}^{\prime}$ \\ Jonathon Colby Gable ${ }^{2}$ \\ Rebekah Zincavage' \\ Gregory L Price (D) ${ }^{2}$ \\ Collin Churchill ${ }^{2}$ \\ Emily Zhu ${ }^{2}$ \\ Keri Stenger $\mathbb{D I}^{2}$ \\ Mukul Singhal' \\ Bal Nepal \\ Michael Grabner (D) \\ Michael J Fisch $\mathbb{D D}^{3}$ \\ David Debono (iD) ${ }^{4}$ \\ Amy R Geschwender ${ }^{5}$ \\ Gebra Cuyun Carter (iD ${ }^{2}$ \\ 'HealthCore, Inc., Wilmington, DE, USA; \\ ${ }^{2}$ Eli Lilly and Company, Indianapolis, IN, \\ USA; ${ }^{3}$ AIM Specialty Health, Chicago, IL, \\ USA; ${ }^{4}$ Anthem, Inc., Indianapolis, IN, \\ USA; ${ }^{5}$ SWOG Cancer Research \\ Network, San Antonio, TX, USA
}

Correspondence: Judith J Stephenson Tel +I 302-547-5770

Email JStephenson@healthcore.com
Purpose: To describe patients' perspectives on the use of and potential challenges and barriers with adherence/persistence to cyclin-dependent kinase 4 and 6 inhibitors (CDK4\&6i's) to treat metastatic breast cancer (MBC).

Methods: This qualitative study consisted of 60-minute semi-structured telephone interviews with patients with MBC in the US who were either current or recent CDK4\&6i users, identified from administrative claims of survey-eligible commercial and Medicare Advantage patients in the HealthCore Integrated Research Database between November 1, 2018 and November 1, 2019. Patients were recruited by email and/or mailed letter. The 60 -minute telephone interviews were conducted by a trained facilitator using a study-developed interview discussion guide that included topics impacting treatment choice and adherence/persistence. Interviews were audio-recorded, transcribed, and thematically analyzed.

Results: All 462 eligible patients were sent a recruitment email and/or letter to which 36 patients responded, consented to participate, and met study inclusion criteria; 25 patients scheduled interviews, and 24 completed them. Study participants were predominately white, non-Hispanic (96\%) with a mean age of 59.5 years. Participants reported a largely positive experience and mentioned very few adherence/persistence issues. They further reported appreciating the ease and convenience of oral oncolytics, coped with side effects, had strong medical and social support, and experienced few cost issues.

Conclusion: The few adherence/persistence issues reported by participants contrasts with other findings of suboptimal oral oncolytic use. Interview themes indicated several factors that likely contributed to the lack of adherence/persistence issues: trusted relationship with oncologist, belief in importance of medication, positive medication views, strong medical and social support, and minimal personal drug cost. Future research should focus on whether and how much these factors impact adherence/persistence in more diverse populations. If adherence/persistence issues are identified in these populations, then it would be appropriate to study the development of interventions that target factors associated with better adherence/ persistence.

Keywords: oral oncolytics, patient perspective, semi-structured interviews, adherence, persistence, CDK4\&6i users

\section{Background}

Breast cancer is the most common non-skin cancer in women, representing $30 \%$ of all new cancer cases among women in the US. ${ }^{1}$ Among patients diagnosed with breast cancer at stages I-III, approximately $30 \%$ progress to stage IV or metastatic breast cancer $(\mathrm{MBC}){ }^{2}$ which has a five-year survival rate of approximately $27 \%{ }^{1,3}$ 
The prevalence of MBC among US women in 2020 was projected to be $168,292 .{ }^{4}$ While treatable, MBC cannot be cured. Depending on the MBC subtype, recommended treatment modalities may include surgery and radiation, as well as hormone therapy, chemotherapy, targeted therapies, or some combination of these therapies. ${ }^{5}$

Targeted cancer therapies are designed to interfere with specific oncogenic molecular pathways and have demonstrated significant clinical activity in MBC, often with a more favorable toxicity profile than conventional cytotoxic chemotherapy. ${ }^{6,7}$ While many therapies have been developed to target human epidermal growth factor receptor 2 (HER2), these therapies are only appropriate for the approximately $20 \%$ of patients with breast cancer that is HER $2+{ }^{8}$ Cyclin-dependent kinase (CDK) 4 and 6 inhibitors (CDK4\&6i's) are a class of targeted therapies for the treatment of hormone-receptor-positive $(\mathrm{HR}+)$, HER2MBC, the most common breast cancer subtype constituting approximately $68 \%$ of female breast cancers. ${ }^{3}$ The first CDK4\&6i (palbociclib) was approved for the treatment of HR+/HER2- MBC in the US in February 2015, followed by ribociclib and abemaciclib in 2017 .

CDK4\&6i's are a type of oral oncolytic, which some patients prefer over injections and infusions for reasons including convenience, increased sense of control, and reductions in injection-site complications. ${ }^{9}$ While oral oncolytics offer some advantages, patient selfadministration of oral therapy shifts the medication management from healthcare providers to the patient, posing potential concerns regarding patients' ability and willingness to follow a prescribed regimen as directed by their healthcare provider (adherence) ${ }^{10}$ or take them for the proper duration (persistence). ${ }^{11}$ Several studies have examined the factors influencing medication adherence/ persistence to oral oncolytics. These studies have had variable results and have identified a need for developing better interventions and measures for assessing adherence/ persistence to these types of oral drugs. ${ }^{12-16}$ Factors that have been consistently identified in the literature as associated with low adherence/persistence include either olderor younger-aged patients, the influence of therapy-related side effects, depression, polypharmacy, higher costsharing, and lack of social support. ${ }^{12,17-20}$ However, much of the literature concerning factors associated with low adherence/persistence in breast cancer has focused on the use of oral oncolytic drugs in general, rather than CDK4\&6i's specifically, and their use as adjuvant therapy to lower the risk of cancer recurrence, as compared to treating metastatic disease. More recently, several metaanalyses of randomized clinical trial data have shown that the addition of CDK4\&6i's to the use of endocrine therapy significantly increased the survival among patients with HR+/HER2- MBC. However, use of CDK4\&6i's was also associated with increased rates of adverse events (AEs) including neutropenia, leukopenia, and diarrhea, which could impact the adherence/persistence to these therapies. $^{21-24}$

Limited evidence exists on real-world adherence/persistence in the CDK4\&6i drug class. One study of 293 commercially-insured patients taking palbociclib found $18 \%$ non-persistence over just 2 months of follow-up. ${ }^{25}$ Additionally, little is known about patients' perspectives regarding the use of these novel oral agents. The purpose of the current study was to learn more about MBC patients' treatment experiences with oral cancer medications and identify factors that influence their medication adherence/ persistence behavior.

\section{Methods}

\section{Data Source and Patient Population}

The HealthCore Integrated Research Database (HIRD) was used as the sampling frame to identify eligible patients from their administrative claims data. The HIRD consists of a broad and geographically diverse spectrum of longitudinal medical and pharmacy claims data from health plan members across the US. The patient population consisted of female patients, aged 18 years or older, who had at least two medical claims with an ICD-10-CM diagnosis code for breast cancer (ICD-10-CM diagnosis codes starting with C50, Z85.3, D49.3), at least one medical claim with an ICD-10-CM diagnosis code for metastases (ICD-10-CM diagnosis codes starting with $\mathrm{C} 77, \mathrm{C} 78$, C79, C80.0, C7B), and at least one pharmacy claim for a CDK4\&6i (abemaciclib, palbociclib, or ribociclib) between November 1, 2018 and November 1, 2019. Patients were also required to be survey-eligible and current health plan members with commercial or Medicare Advantage health insurance.

\section{Recruitment Process}

A purposive sample of 462 eligible patients was identified and all 462 eligible patients received recruitment materials with a target of 25 completed interviews. This target was based on the size of the available sample, an estimate of the number of completed interviews that could be obtained 
from the sample, and literature that indicated 25 interviews in a homogeneous population should be sufficient to reach saturation. $^{26}$ Patients received a recruitment email (as available), followed by a mailed letter, and lastly a telephone call (as available) if they did not respond to the initial outreach. Recruitment continued until 25 telephone interviews had been scheduled at which time the study closed. A maximum of 5 attempts was made to contact patients, after which no further attempts were made.

The recruitment email and/or letter informed the patient about the study, including how and why they were identified for participation, what participation entailed, as well as the compensation they would receive if they completed the telephone interview. It also included a phone number to call to see if they qualified (ie met study inclusion criteria) and to schedule the 60-minute telephone interview, and a telephone number and email address to contact HealthCore to obtain more information about the study or to be placed on the Do-Not-Contact list.

Patient qualification consisted of verifying their name, month and year of birth, and current health plan membership, as well as confirming their diagnosis of MBC and current or recent use of a CDK4\&6i. Patients were also required to be able to complete the interview in English. Patients who were no longer interested in the study or failed at least one of the study inclusion criteria were excluded and not contacted again about the study. Patients who qualified and met all screening criteria were directed to the online informed consent form that they had to read, sign electronically, and save before they could schedule the interview.

\section{Data Collection}

Data were collected via semi-structured telephone interviews conducted by an experienced facilitator who was trained on the study protocol and discussion guide. The same facilitator conducted all interviews using the studydeveloped discussion guide.

At the beginning of the scheduled interview call, the facilitator went through an introduction that stated the purpose of the interview was to talk about participants' experiences with oral cancer pills, defined as drugs taken by mouth and swallowed to treat their cancer. The introduction reminded participants that their participation was voluntary, anything said was confidential, and information would be reported in a way that protected participants' privacy. The facilitator also repeated key items from the informed consent document, and administered the short pre-questionnaire consisting of demographic and clinical questions that were used to profile participants. Participants were informed the interview discussions would be audio-recorded for analytic purposes and redacted transcripts would be shared with the study sponsor. Participants could stop the interview and resume at another time if they asked to do so. The interview discussions lasted approximately 60 minutes and patients who completed the interview received a check for $\$ 200$ to compensate them for their time.

\section{Interview Discussion Guide}

The interview discussion guide was developed with input from all authors and consisted primarily of guided openended questions and prompts for the facilitator. It was decided that the term oral cancer pills, defined as drugs that are taken by mouth and swallowed to treat their cancer, would be defined at the beginning of the interview, and used as a proxy for CDK4\&6i medication use during the interview since current or recent use of a CDK4\&6i medication was a required study inclusion criterion.

The discussion guide served as a "road map" and memory aid for the facilitator; it reminded the facilitator of the interview topics to be covered while allowing flexibility in the order the discussion topics were covered. The facilitator was free to apply probes when needed to elicit additional detail or seek clarification regarding the patient's response; however no specific probes were included in the interview guide. (See Supplemental File for interview discussion guide.)

The primary objective of these interviews was to better understand the perspectives of women with $\mathrm{MBC}$, who were either current or recent CDK4\&6i users, concerning their experiences with these oral treatments and the impact of their experiences on medication adherence/persistence. Four major topics were discussed: The patient's experience with their MBC diagnosis and treatment decision-making; their experiences and concerns surrounding CDK4\&6i use especially as it related to adherence/persistence; their interactions and experiences with providers, other healthcare professionals, and the healthcare system around the use of CDK4\&6i's; and their reflections and viewpoints on what could be done differently to better prepare or help patients regarding their diagnosis of $\mathrm{MBC}$, treatment options, and the use of CDK4\&6i's. 


\section{Data Analysis}

Demographic and clinical characteristic data were tabulated from the pre-questionnaires and presented as a patient profile in aggregated form.

The audio recordings of the interviews were transcribed, and a qualitative descriptive analysis of the interview discussion data was performed that used the responses of all participants to structure the dialogue, identify common themes of interest, and present quotes to support the themes. Since questions were open-ended, participants responded in different ways, and in order to ensure patient privacy language was used to convey whether specific themes or sentiments were shared among the entire group of respondents or a larger/ smaller portion of the group. This was done in the form of statements such as "a few" or "the majority"; more specific terms such as "approximately a third" or "roughly half" were also used depending on the heterogeneity of the responses.

\section{Human Subject Protection}

As protected health information (PHI) was required for the conduct of this study, a waiver of authorization was applied for and obtained from an Institutional Review Board. All study materials, including the recruitment email/letter, recruiting script, informed consent, study protocol, and discussion guide were submitted to and approved by the New England Institutional Review Board (NEIRB\#: 120190520) prior to the commencement of data collection activities, and all patient data were handled in compliance with the regulations of the US Health Insurance Portability and Accountability Act (HIPAA) of 1996. Participants were required to provide electronic consent to an online informed consent form before they were able to schedule their interview. The informed consent form included a statement that participants' anonymized responses might be published in medical journals or shared with others as part of scientific discussions. Electronic consent meant that patients read the form, checked the box acknowledging consent to participate in the study, typed their name and date indicating their consent to participate and saved/submitted the form. Finally, at the beginning of participants' scheduled interviews, the facilitator repeated key items from the informed consent document, and asked participants to confirm once more that they were still willing to participate in the interview.

\section{Results}

Of 462 eligible patients who received recruitment emails and/or letters, 57 responded to the recruitment email/letter or were contacted by an interviewer over the telephone; 36 patients were screened and met study inclusion criteria; 25 patients completed the online informed consent form and scheduled interviews; and 24 patients completed the 60minute telephone interview between January and February 2020 (Figure 1).

The participants had a mean (standard deviation (SD)) age of 59.5 (9.91) years, 23 were white/non-Hispanic (95.8\%), 19 were married/had domestic partner (79.2\%), and 22 had at least some college education (91.7\%). A high proportion of participants reported use of alternative/complementary medicines $(87.5 \%)$ and oral prescription medications for other conditions (79.2\%) (Table 1).

Results of the interviews were organized around specific themes within each of the four guiding topics that characterized participants' experiences with oral cancer pills and their impact on medication adherence/persistence.

\section{Theme I: MBC Diagnosis and Treatment Decision-Making}

Patients reported varied pathways to their MBC diagnosis that often involved multiple health care professionals (HCPs) before their oncologist. Some patients were initially seen by a primary care physician, urgent care center, or an emergency department for seemingly unrelated symptoms that led to further tests "that started the ball rolling." [Patient A11]

Most patients expressed "surprise" and "shock" at the time of their MBC diagnosis, especially those who had been cancer-free for a long time or whose current symptoms seemed unrelated to breast cancer. "My original breast cancer was in 2002 ... I thought we were pretty far along. It was never my expectation that I was going to receive a metastatic diagnosis." [Patient A6]

A few patients, specifically one with ongoing medical issues since her initial breast cancer diagnosis and another with a family history of MBC, were not surprised. For most patients, the biggest fears at diagnosis were imminent death or facing the prospect of receiving intravenous (IV) chemotherapy again. A few said they would not do IV chemotherapy again and wondered what treatment options they would have. "I didn't want chemo. I went through that in the 90's and was not going to do that again." [Patient A2]

Most patients reported that having a trusted relationship with their oncologist was of utmost importance to them as they primarily relied on their oncologists' recommendations to make their treatment decisions. 


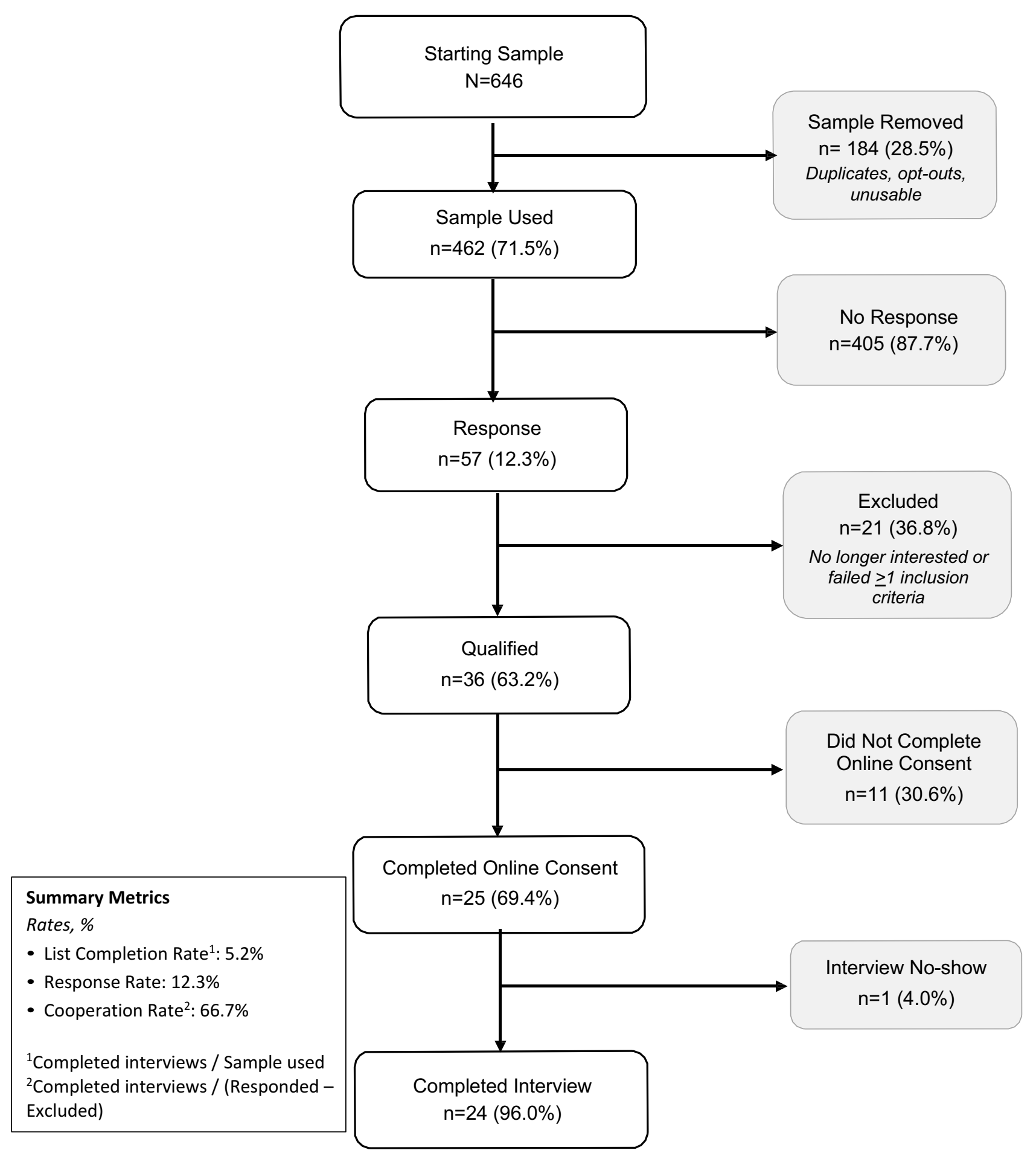

Figure I Sample Disposition.

I have known my oncologist for a long time and I obviously have a choice, but I do trust her. I guess at that point I wasn't even really looking for any other option. [Patient A17]
While most patients reported having a trusted relationship with their oncologist, a few who did not reported looking for a new oncologist. 
Table I Participant Characteristics

\begin{tabular}{|c|c|c|}
\hline \multirow[t]{2}{*}{ Characteristic } & \multicolumn{2}{|c|}{$\begin{array}{c}\text { Participants } \\
\text { N }=24\end{array}$} \\
\hline & n/Mean & $\% / \mathbf{S D}$ \\
\hline Age in years, mean, SD & 59.5 & 9.91 \\
\hline $\begin{array}{l}\text { Race/Ethnicity, n, \% } \\
\text { White, non-Hispanic }\end{array}$ & 23 & $95.8 \%$ \\
\hline $\begin{array}{l}\text { Marital Status, n, \% } \\
\text { Married/Domestic partner }\end{array}$ & 19 & $79.2 \%$ \\
\hline $\begin{array}{l}\text { Children/Grandchildren }<18 \text { Years Living } \\
\text { in Household (Yes), n, \% }\end{array}$ & 5 & $20.8 \%$ \\
\hline $\begin{array}{l}\text { Education, } \mathbf{n}, \% \\
\text { At least some college, college graduate }\end{array}$ & 22 & $91.7 \%$ \\
\hline $\begin{array}{l}\text { Current Job Status, } \mathbf{n}, \% \\
\text { Employed full-/part-time outside/at home } \\
\text { Unemployed, retired, other }\end{array}$ & $\begin{array}{c}9 \\
15\end{array}$ & $\begin{array}{l}37.5 \% \\
62.5 \%\end{array}$ \\
\hline $\begin{array}{l}\text { Job Status at Time of MBC Diagnosis, n, \% } \\
\text { Employed full-/part-time outside/at home } \\
\text { Unemployed, retired }\end{array}$ & $\begin{array}{c}16 \\
8\end{array}$ & $\begin{array}{l}66.7 \% \\
33.3 \%\end{array}$ \\
\hline $\begin{array}{l}\text { Job Status Changed Because of MBC } \\
\text { Diagnosis (Yes), n, \% }\end{array}$ & 9 & $37.5 \%$ \\
\hline $\begin{array}{l}\text { Reasons for Change in Job Status, } \mathbf{n}, \% \\
\text { Symptoms and side effects; feeling bad; pain- } \\
\text { related; medical leave related to chemo and } \\
\text { radiation treatment } \\
\text { To reduce stress levels } \\
\text { Husband sick; decided time to retire } \\
\text { More work from home }\end{array}$ & $\begin{array}{l}3 \\
1 \\
1\end{array}$ & $\begin{array}{l}33.3 \% \\
11.1 \% \\
11.1 \%\end{array}$ \\
\hline $\begin{array}{l}\text { Use Alternative/Complementary } \\
\text { Medicines (Yes), n, \% }\end{array}$ & 21 & $87.5 \%$ \\
\hline $\begin{array}{l}\text { Number of Complementary/Alternative } \\
\text { Medicines Used, mean, SD }\end{array}$ & 3.0 & 1.94 \\
\hline $\begin{array}{l}\text { Take Oral Prescription Medications for } \\
\text { Other Conditions (Yes), n, \% }\end{array}$ & 19 & $79.2 \%$ \\
\hline $\begin{array}{l}\text { Typical Day, Number of Oral Prescription } \\
\text { Medications Taken, mean, SD }\end{array}$ & 3.8 & 2.18 \\
\hline $\begin{array}{l}\text { Typical Day, Number of Oral OTC } \\
\text { Medications Taken, mean, SD }\end{array}$ & 3.0 & 2.25 \\
\hline $\begin{array}{l}\text { How Receive Oral Medications and Oral } \\
\text { Cancer Pills, n, \% }\end{array}$ & & \\
\hline Mail order only & 8 & $33.3 \%$ \\
\hline Big box, independent or chain pharmacy only & 3 & $12.5 \%$ \\
\hline Both & 13 & $54.2 \%$ \\
\hline
\end{tabular}

Abbreviations: SD, standard deviation; MBC, metastatic breast cancer; OTC, over the counter.
My first oncologist, I didn't like her attitude ... I felt defeated, and I need someone who is more hopeful ..., so I found a new oncologist ... more hopeful, more optimistic. [Patient A22]

A few other patients even switched oncologists multiple times. Some patients sought specialists in renowned cancer centers because the reputation of these centers gave patients higher confidence in their oncologists and the care they received.

While patients recalled having discussions with their oncologists regarding whether to initiate oral treatments, most patients did not recall discussing options regarding the use of a specific oral treatment. "I didn't have an option for A, B or C. I could have said 'no' but they didn't say 'do you want to do this or that?" [Patient A11] Initially, very few patients were aware that there was more than one oral treatment option, and those that were aware deferred to their oncologist to decide the best treatment. "It wasn't much of a discussion of options. It was 'this is the gold standard, this combination, so this is what we are going to do." [Patient A16] A few patients also sought second opinions prior to starting treatment. Most of those patients who sought second opinions found they aligned with the initial treatment recommendation. One patient sought opinions from three different oncologists who all agreed with the original treatment decision.

Almost all patients reported the initial conversation covered treatment expectations and potential side effects and often included a nurse practitioner who was able to spend additional time reviewing treatment details and answering questions. For a few patients, the specialty pharmacist was also involved. The main message almost all patients remember receiving was that while $\mathrm{MBC}$ is not curable, it can be treated, and while medications can have side effects, these too can be treated. Many patients initially focused on outcomes, and therefore details regarding side effects were vague. When side effects were mentioned, patients reported being informed about low white blood cell counts, being more prone to infections, diarrhea, nausea, joint and muscle pain, and fatigue. Some patients were assured of being able to combat side effects, with their physician either detailing management protocols in advance or reassuring patients that side effects could be managed as they occurred. 
He basically told me that this should continue to work and basically kill the cancer and we use it for a good three years or better ... what side effects he mentioned never happened for me. [Patient A2]

"He mentioned that there were some bad intestinal, like diarrhea, but they are aware of it and have a protocol in place." [Patient A7]

He talked about side effects and symptoms but more about that the drug can delay the progression of the cancer. That I can live with the side effects without too much trouble. [Patient A4]

Once the oral treatment decision was made, no patients reported that their oncologist raised the subject of adherence/persistence. Several patients indicated they assumed the importance of adherence/persistence to the oral treatment without any discussion - "I didn't question it." [Patient A9]. Due to their previous cancer experience, these patients understood the importance of taking the medication as prescribed to keep their cancer from progressing.

I don't think they (oncologist) needed to [talk about the importance of adherence]. It was understood this was the only thing that was going to keep the cancer at bay. This is it; this is what you've got to do. It was clear that it was important, it's not necessary to say that. [Patient A16]

One patient said, "Well, when you prescribe a medication you expect the person to take it when they're supposed to." [Patient A2].

\section{Theme 2: Experiences with Oral Cancer Medications}

When asked, all patients reported the length of time they took their current $\mathrm{MBC}$ oral cancer medication. Responses ranged from 2 months to 4 years with an average of 14 months. Overall, most patients reported their experiences with oral treatment were positive and preferable to previous IV chemotherapy. "I like anything that doesn't involve a needle." [Patient A24] Effectiveness was reported to be the biggest benefit with most patients stating the effectiveness of their oral cancer medications made it easier for them to tolerate side effects because their cancer was not progressing. "I'm having side effects but what's more important? Do you want to go to your granddaughter's graduation or lay in bed?" [Patient A18]

Approximately half of the patients reported their overall experience with oral cancer medications was more positive than negative, citing such reasons as ease of use (taken at home, no schedule disruption, no needles), effectiveness (drop in disease activity biomarker levels, stable CT scan), and fewer, less severe side effects, especially hair loss and nausea. Most of the other patients' experiences were neutral; these patients commented that while the treatment had a positive impact on controlling their cancer, side effects impacted their everyday life and consistent medication use was difficult due to low white blood cell counts disrupting medication use and frequent dosage changes. The few patients whose experiences were more negative than positive cited problems with side effects, including tiredness, fatigue or low stamina, low white blood cell count, being prone to infections, nausea or diarrhea, dry eye, and not being able to eat grapefruit. However, almost all patients agreed that oral treatment was "easier" and preferable to IV chemotherapy. "Seems to be working. It's easier than chemo. You get a good response and a good quality of life." [Patient A3] "It's easier. You don't have to go in. It's a much milder treatment and one can function." [Patient A12] "I get good scans so they must be doing their job. It's simple, easy to take." [Patient A12]

While the importance of taking their medication at the same time each day was stressed, other details were vague leaving patients to work out the details of their routines themselves based on trial and error, eg:

I have no idea when I am supposed to take it. It's easier for me to remember to take them in the morning. I may have researched with or without food and I don't think it mattered. I take them before I've eaten anything. [Patient A16]

We may have had a conversation about that, but I am not $100 \%$ sure. I think the best time of day to take my medication is the time at which I will remember to take my medication. [Patient A6]

Patients reported few adherence/persistence problems. A handful of patients mentioned unintentionally missing a dose or two at the beginning of treatment, before they adjusted to their new regimen and a few other patients reported temporarily discontinuing them for upcoming medical procedures, illnesses such as a cold or flu, or to reduce fatigue or infection risk during vacations, travel, or family events. A few others stated they occasionally took a dose later than intended, but reminders or visual cues kept them from missing the dose all together. Pill organizers or an alarm on their phones were mentioned as effective reminders to track their medication use. Most patients reported having 
a routine that helps with adherence/persistence, such as keeping the pills in a place that serves as a visual reminder or taking their pills at a consistent time each day. Only a very few patients reported forgetting or intentionally skipping their medication without first checking with their oncologist.

The main motivation for medication adherence/persistence was simply wanting to stay alive and patients viewed taking them as directed tied to success. Patients also expressed wanting to keep cancer controlled so they could continue to live as normally as possible, including continuing to raise children or attending family milestone events such as graduations or weddings. "If you don't take this stuff, you're probably going to die a lot sooner. The motivation is to stay alive as long as possible." [Patient A12] "I have a grandson that lives with us ... he has lived with us since he was five, so my motivation is to see him fully graduate from college." [Patient A11]

While intentionally skipped doses were rare, oncologist decisions to change dose or interrupt the treatment regimen were common and typically due to low white blood cell/neutrophil counts. About half of the patients reported experiencing delays in resuming treatment at the end of a cycle for this reason, and slightly fewer reported a change in dose. For instance, one patient indicated:

I was on two months then immediately changed. Now I am on $100 \mathrm{mg}$ and have changed two times, or maybe more. I think she is considering going down even more if I have to wait another week to start. [Patient A22]

About half the patients who experienced a treatment interruption reported it happening multiple times.

I've gone off a couple of times where I was off for a month so things would get back to a satisfactory level. I changed the dose about 3 months into it, ..., but still having to extend breaks after the reduced dose. [Patient A3]

For a few of these patients, oncologists altered dosing to unusual treatment schedules such as two weeks on, two weeks off, or every other day.

While the impact of oral oncolytic use on patient lifestyle varied greatly, the majority of patients reported having to make some lifestyle changes to manage side effects. The primary lifestyle impacts associated with side effects were lack of stamina, gastrointestinal issues, and the need to avoid crowds due to a higher risk of infection associated with oral oncolytic use. Most patients reported still being able to participate in desired activities on a limited basis and fulfill their daily living activities.
I slowed down more, more tired. I'm doing less because I don't have the energy. Also, I have joint pain and get out of breath easily. I still drive and go places, meet friends for lunch, cook dinner, vacuum but I have to pace myself. [Patient A4]

Only a very few patients mentioned a direct impact of their treatment on their ability to work with a few transitioning to more work at home or shorter hours.

I'm extremely conscientious of germs and staying well. I officially stopped working in July 2019 to reduce stress and be able to reduce exposure to germs. I don't shake hands or use others' pens. [Patient A6]

\section{Theme 3: Interactions and Experiences with Providers, Healthcare Professionals, and the Healthcare System}

For the most part, patient interactions and experiences with healthcare professionals and the healthcare system were positive. Almost all patients said they had support from their oncologist's office, often from the office staff. For some patients, this was a result of changing oncologists to find a more supportive relationship. The types of physician support mentioned by patients included easy access to providers through portals such as MyChart or monitored voice mail systems, quick responses to questions, depth of staff to respond to inquiries, and a triage system to answer urgent questions quickly.

They have an online chart where I can ask questions ... either the oncologist or the physician assistant (PA) can answer. The PA gets back to me sooner or I can also talk with the nurses ... she can ask the doctor then get back to me. [Patient A23]

A few patients saw an oncologist in a major cancer center, that offered increased support and staff including social workers, pampering days, wig resources, and support groups. A few offices had a patient navigator to serve as a point-person for patients. One patient even mentioned she missed the interaction with and information from nurses and social workers from her previous IV chemotherapy and wondered what information she was missing not being in that environment since that was when the social worker met with patients.

Most patients described the medication refill process as "easy", although challenges occurred when there were changes in insurance or specialty pharmacy, something 
several patients experienced. A few patients found having to set up one's prescription within a new system to be time consuming and confusing, even when they were notified of the change by their insurance provider ahead of time. A couple of patients said they have had difficulty registering, believing they had completed the process only to find that there was information missing or they had not registered at all. Two patients indicated changes and missing information caused refills to be delayed by a few days (on one occasion each).

Patients indicated that the specialty pharmacy plays an important role in providing information and helping patients stay on track with prescriptions. Some patients mentioned being able to reach out to the specialty pharmacy easily with questions. "I keep the number for the pharmacy in my phone so if I ever have any questions, I can call them." [Patient A8] For most patients, the monthly contact with the specialty pharmacy for refills also served as a reminder for taking the medication appropriately.

They scare you to death every time you get your prescription. You speak with a pharmacist and they tell you don't stop it, don't double up, don't eat grapefruit. [Patient A20]

While patients were aware of the high cost of their medication, most indicated they pay nothing or only a small co-payment due to insurance and co-payment assistance from foundations or pharmaceutical companies. Only one patient indicated that paying for her oral oncolytic was a financial hardship. Patients were often not aware of the details of how assistance worked because they received help from medical assistants in their doctor's office or cancer center. "I am not paying anything. I have a discount. I don't know how it works but I have no copay." [Patient A4]

\footnotetext{
My insurance covered most of the expense. Then there was an organization that contacted my insurance company ... they paid all the parts I was expected to pay. I don't know how that started. [Patient A9]
}

Despite the reported low current cost burden of their treatment for them, most patients were concerned about the overall cost of health care and medication costs in the future. While cost has not had a negative impact on adherence/persistence for this group of patients, most patients said they did not know how they would pay for their medication if they lost their insurance, experienced a change in coverage, or if they lost assistance with copays. "I am nervous because I don't know what's going to happen with cost now that I am on a new plan. I don't know what it is going to cost me." [Patient A8] A few patients added that they would stop their medication if it became a financial hardship rather than put their family in a difficult financial situation. "I get the insurance through my husband's employer. If he would retire ... we do think about how we would pay for it." [Patient A17]

\section{Theme 4: Reflections on What Could Be Done Differently to Better Prepare Patients Regarding Their MBC Diagnosis and Treatment Options}

Many patients indicated that knowing more about MBC, the next steps, and the overall treatment pathway would have helped them feel more prepared at the start of their MBC journey. Initially, these patients said that their trust and confidence in their oncologist along with the materials and conversations about their treatment made them feel confident and prepared for their treatment. Upon probing, these patients also mentioned several ways to improve patient preparedness, including more education about MBC and its symptoms. Most patients were unaware of symptoms and "what to look for." Some patients wondered how treatment and prognosis could have been impacted with earlier detection. There should be "More awareness about what to look for ... What if mine could have been caught earlier?" [Patient A3]

Some of these patients also wanted to know more about the options and next steps. Although most patients were very satisfied with the treatment plan laid out, some wondered "what's next?"

When you get to stage IV you're in a totally different class. What worked before may not work, options are different. Having the different protocols outlined for you is very important. It would be great if there was information ... for all stage IV people ... a conference, meeting, here is what there is available to help you. [Patient A7]

While knowing more about other options may have little impact on the initial treatment decision, understanding more about the overall process would bring comfort to some patients.

In the future, as more options become available, some patients felt it important to find a way for patients to better 
understand treatment differences and guide them through their options.

Seems as time goes on there are options. People may need an interested third party to help guide them through the options ... At the time I went on I don't think there were options but someone walking through the options and the side effects and finances would be huge. [Patient A20]

Support from family and friends through listening, expressing love and appreciation, and helping with household tasks or medical visits was viewed by all patients as important and meaningful. All patients reported that they get adequate support from family and/or friends with the level of support depending on the needs of the patient. "They want you to know they are there for you. No one would leave me alone. They text message, call, and continue to check in when things quiet down." [Patient A8]

Only a few patients participated in support groups, and those that did appreciated connecting with others in a similar situation to share information, support, and hope. Patients that did not participate in support groups cited perceived negativity or trouble finding groups with patients who were similar to them. Instead, some patients sought private support through a therapist or counselor, participated in conferences focused on education and support, or participated in active community or fund-raising events such as a $5 \mathrm{~K}$ walk.

All patients were upbeat in their advice offered to other patients regarding the use of oral oncolytics - "Try it because there is no harm in trying oral therapy to see how it works!" "If you have bad side effects talk to your doctor ... don't pretend that your quality of life hasn't been hurt. There are things that can be done." [Patient A24] More advice from these patients was to do as your doctor says and take it consistently, do not skip doses, take it every day and at the same time every day. That is the only way to know for sure whether it is working. "Be honest with your doctor because they can do things like lower the dose. Or change the frequency." [Patient A14]

\section{Discussion}

Overall, the 24 participants reported a positive experience with their CDK4\&6i oral cancer pills. Participants generally did not view drug adherence/persistence as an issue for them. They reported taking their medication according to their oncologists' instructions, including following oncologist-ordered medication interruptions and dosing changes. Most participants appreciated the ease and convenience of oral cancer pills, were able to cope with side effects, had strong medical and social support, and were not currently experiencing cost issues. The importance of a trusted relationship with their oncologist was emphasized by nearly all participants.

While estimates of oral oncolytic adherence/persistence rates reported in the literature vary substantially, from $23-100 \%,{ }^{13,14,16,17,27}$ due to differences in patient populations, tumor types, medication, follow-up period, and lack of a standard measure of adherence/persistence,$12,14,16,28$ suboptimal adherence/persistence to oral oncolytics is a common concern. Given the relatively recent introduction of CDK4\&6i's, real-world adherence/persistence estimates for this specific drug class are limited. The lack of adherence/persistence issues reported by study participants contrasts with a recent retrospective claims study of a larger population of CDK4\&6i users identified from pharmacy claims data in the HIRD where only $58 \%$ of patients were classified as adherent (using proportion of days covered (PDC) $\geq 0.80$ ) and $23 \%$ of patients discontinued within a 6 month period (with a permissible gap of up to 60 days). ${ }^{29}$ However, this claims-based analysis could not account for oncologist-ordered delays in treatment or altered dosing schedules due to low white blood cells, which about half of the current participants reported. Also, while some oral oncolytics are used as adjuvant therapy for breast cancer, this study focused on the use of oral agents for advanced metastatic disease where the purpose and evaluation of the effect of the therapy may be different.

While this may account for some of the observed difference, the disconnect between the lack of patientreported adherence/persistence issues reported by study participants versus the lower claims-determined adherence/persistence rates reported in literature may be due in part to several beneficial factors reported by study participants and the self-selection of these patients for the interviews. In a recent systematic review of psychosocial motivators and barriers of oral oncolytics for breast cancer, the top two best-supported factors associated with higher adherence/persistence were patient-physician relationships and belief in the importance and benefit of the therapy, ${ }^{18}$ each of which was reported by most study participants. Most participants reported the importance of a trusting relationship with their oncologist, and a few actively sought a different oncologist when they felt they did not have that supportive relationship. A strong patientprovider connection also enables greater patient 
knowledge of their cancer, medication, and side effects, which further increases adherence/persistence. ${ }^{18}$ This is consistent with themes identified by study participants who described strong medical support and information sharing from the oncology office, especially support staff, easy provider access through patient portals or monitored voice mail systems, as well as monthly contact and opportunity for ad hoc questions with the specialty pharmacy.

Most participants were confident that their treatment would keep their cancer in control and were highly motivated. Because this was a metastatic/advanced population, many participants had previously received breast cancer treatments, and indicated the importance of medication adherence/persistence as they now faced advanced disease. Participants frequently reported that their medication was vital to their survival and expressed not wanting to miss specific family experiences or life events.

Prior studies report that treatment-related side effects are dominant factors associated with non-adherence/persistence to oral oncolytics. ${ }^{12-14,30}$ However, positive views of the medication and ability to cope with symptoms are associated with increased adherence/persistence, ${ }^{18}$ which is consistent with experiences reported by participants. Participants reported their oral medications were easier to use and had more manageable side effects, especially compared to their previous experiences with IV chemotherapy. Most participants also mentioned that their cancer was not progressing, which made it easier to tolerate side effects.

Cost is often associated with decreased adherence/ persistence, ${ }^{12,14,17,30}$ but despite the cost of CDK4\&6i's, ${ }^{31}$ most patients in this sample paid either very little or nothing due to a combination of insurance and co-payment assistance. Most participants knew very little about co-payment assistance as these details were handled by oncology support staff. This could serve as a barrier for patients without access to this level of support, as patient financial assistance programs are associated with a significant administrative burden. ${ }^{32,33}$ These programs are prevalent in oncology and estimated to cover $87 \%$ of all FDA-approved oncology drugs in the US, ${ }^{33}$ but broad patient usage statistics for these programs are not known, nor are usage statistics specific to MBC. In retrospective studies in single cancer care institutions, usage estimates range from $12 \%$ of all oral oncolytic prescriptions $^{34}$ to $32 \%{ }^{35}$ and $36 \%{ }^{36}$ of oncology patients filling prescriptions for oral oncolytics at the specialty pharmacy.

Self-efficacy in treatment decision-making, administration, and management is also consistently associated with medication adherence/persistence. ${ }^{18,30}$ Most patients deferred to their oncologist with whom they felt a trusted connection to determine the specific treatment medication. In contrast, participants described managing their drug regimen by developing a routine for taking their medication that worked best for them in terms of visual or digital reminders and side effects (eg, with/without food, time of day) on their own without much input from their HCPs. The literature suggests that higher levels of selfefficacy are associated with lower levels of both intentional and unintentional non-adherence/persistence. ${ }^{30}$ Indeed, when probed about how HCPs could have better supported them when starting oral oncolytics, participants reported themes that focused on areas that facilitate selfefficacy: educating patients about $\mathrm{MBC}$ and symptoms ahead of diagnosis, providing an overall view of the treatment pathway to help patients know what to expect, and helping patients understand medication differences as more oral oncolytics for MBC become available.

\section{Limitations}

While this qualitative study adds important first-hand patient perspectives on factors affecting adherence/persistence to oral oncolytics in $\mathrm{MBC}$, some limitations should be noted. This research was conducted with a small number of predominantly (96\%) white, non-Hispanic participants who responded to our recruitment materials and consented to participate in the study and is not likely representative of the overall population of CDK4\&6i users. Patient experiences in this sample were generally positive and reflected many factors associated with increased adherence/persistence, including strong medical and social support, which might not be as prevalent in a larger or more socioeconomically diverse population. Finally, this sample consisted of patients who were enrolled in commercial or Medicare Advantage health insurance plans in the US and satisfied all inclusion criteria; therefore, these results may not be generalizable to patients who did not respond to the recruitment materials, or who had other types of health insurance (including Medicaid), the uninsured, and health plans outside the US.

As is common in qualitative research, patient responses may be subject to recall bias and social desirability. It is also possible that patients may have overstated their 
positive experiences or over-estimated their own adherence/persistence. A systematic review of studies that compared patient-reported adherence versus objective measures found a strong correlation between the two, but those that directly compared measures found patientreported measures were higher. ${ }^{28}$ We expect this effect to be minimized in the current study as patients were probed about potential experiences and barriers that may have impacted adherence/persistence.

Finally, this qualitative interview study is considered observational and purely descriptive in nature; results should be evaluated to inform future hypothesis generation, and not as definitive conclusions. Because this observational study was conducted with a purposive sample of patients, any characterizations of frequency, such as "a few, many, or most", are unlikely to accurately reflect the experience among all patients who have been treated with a CDK4\&6i for MBC.

\section{Conclusion}

The lack of adherence/persistence issues reported by the 24 participants in this qualitative study of CDK4\&6i use for MBC is inconsistent with findings from other oral oncolytic studies in the literature that report suboptimal adherence/persistence and may be due to differences in patient populations, tumor types, medications, follow-up period, and lack of a standard measure of adherence/ persistence. Themes that emerged from the interviews indicate several factors that likely impacted the lack of medication adherence/persistence issues reported by these patients: trusted relationship with their oncologist, belief in the importance of their medication, positive views of their medication, strong medical and social support, and minimal personal drug cost. Increased education related to $\mathrm{MBC}$ diagnosis and symptom recognition, awareness of the overall treatment pathway, and understanding differences in drugs were identified by participants as areas for improvement. Future research should focus on quantitative assessments of adherence among more generalizable samples of real-world patients as well as mixed method studies. It is hoped that future studies will involve the development of interventions that promote factors associated with high adherence/persistence.

\section{Acknowledgments}

Elizabeth Apgar, Clinical Research Contractor for HealthCore, Inc., provided writing and editorial support for this manuscript. This study was funded by Eli Lilly and Company.

\section{Disclosure}

Gregory L Price, Collin Churchill, and Keri Stenger are employees and shareholders of Eli Lilly and Company. Gebra Cuyun Carter, Jonathon Colby Gable, and Emily Zhu were employees of Eli Lilly and Company at the time the study was conducted. Judith J Stephenson, Mukul Singhal, Bal Nepal, Michael Grabner, and Rebekah Zincavage are employees of HealthCore, Inc., which received funding from Eli Lilly and Company for the conduct of this study. Michael Grabner and Judith J Stephenson are shareholders of Anthem, Inc. Michael $\mathrm{J}$ Fisch is an employee of AIM Specialty Health and parttime faculty at the University of Texas MD Anderson Cancer Center. David Debono is an employee of Anthem Inc. Neither Dr Fisch or Dr Debono received compensation for their participation in this study. Both are shareholders of Anthem, Inc. Amy $\mathrm{R}$ Geschwender is a consultant for the SWOG Cancer Research Network and received no compensation for her participation in this study. The authors report no other conflicts of interest in this work.

\section{References}

1. Siegel RL, Miller KD, Jemal A. Cancer statistics, 2020. CA Cancer J Clin. 2020;70(1):7-30. doi:10.3322/caac.21590

2. O'Shaughnessy J. Extending survival with chemotherapy in metastatic breast cancer. Oncologist. 2005;10(Suppl 3):20-29. doi:10.16 34/theoncologist.10-90003-20

3. National Cancer Institute. SEER cancer stat facts: female breast cancer. Available from: https://seer.cancer.gov/statfacts/html/breast. html. Accessed August 17, 2021.

4. Mariotto AB, Etzioni R, Hurlbert M, Penberthy L, Mayer M. Estimation of the Number of Women Living with Metastatic Breast Cancer in the United States. Cancer Epidemiol Biomarkers Prev. 2017;26(6):809-815. doi:10.1158/1055-9965.EPI-16-0889

5. The American Cancer Society medical and editorial content team. Treatment of Stage IV (Metastatic) Breast Cancer. American Cancer Society; 2020. Available from: https://www.cancer.org/cancer/breastcancer/treatment/treatment-of-breast-cancer-by-stage/treatment-ofstage-iv-advanced-breast-cancer.html. Accessed August 25, 2020.

6. Garcia-Aranda M, Redondo M. Protein Kinase Targets in Breast Cancer. Int J Mol Sci. 2017;18(12):2543. doi:10.3390/ijms18122543

7. Shah NP, Tran C, Lee FY, Chen P, Norris D, Sawyers CL. Overriding imatinib resistance with a novel ABL kinase inhibitor. Science. 2004;305(5682):399-401. doi:10.1126/science.1099480

8. The American Cancer Society medical and editorial content team. Targeted therapy for breast cancer. American cancer society.; 2020. Available from: https://www.cancer.org/cancer/breast-cancer/treat ment/targeted-therapy-for-breast-cancer.html. Accessed August 25, 2020.

9. Borner M, Scheithauer W, Twelves C, Maroun J, Wilke H. Answering patients' needs: oral alternatives to intravenous therapy. Oncologist. 2001;6(Suppl 4):12-16.

10. Spoelstra SL, Given BA, Given CW, et al. Issues related to overadherence to oral chemotherapy or targeted agents. Clin J Oncol Nurs. 2013;17(6):604-609. doi:10.1188/13.CJON.17-06AP 
11. Given BA, Spoelstra SL, Grant M. The challenges of oral agents as antineoplastic treatments. Semin Oncol Nurs. 2011;27(2):93-103. doi:10.1016/j.soncn.2011.02.003

12. Verbrugghe $M$, Verhaeghe S, Lauwaert K, Beeckman D, Van Hecke A. Determinants and associated factors influencing medication adherence and persistence to oral anticancer drugs: a systematic review. Cancer Treat Rev. 2013;39(6):610-621. doi:10.1016/j.ctrv. 2012.12.014

13. Jacobs JM, Ream ME, Pensak N, et al. Patient experiences with oral chemotherapy: adherence, symptoms, and quality of life. J Natl Compr Canc Netw. 2019;17(3):221-228. doi:10.6004/jnccn.2018. 7098

14. Greer JA, Amoyal N, Nisotel L, et al. A systematic review of adherence to oral antineoplastic therapies. Oncologist. 2016;21 (3):354-376. doi:10.1634/theoncologist.2015-0405

15. Sikorskii A, Given CW, Given BA, et al. An Automated Intervention Did Not Improve Adherence to Oral Oncolytic Agents While Managing Symptoms: results From a Two-Arm Randomized Controlled Trial. J Pain Symptom Manage. 2018;56(5):727-735. doi:10.1016/j.jpainsymman.2018.07.021

16. Huang WC, Chen CY, Lin SJ, Chang CS. Medication adherence to oral anticancer drugs: systematic review. Expert Rev Anticancer Ther. 2016;16(4):423-432. doi:10.1586/14737140.2016.1159515

17. Mathes T, Pieper D, Antoine SL, Eikermann M. Adherence influencing factors in patients taking oral anticancer agents: a systematic review. Cancer Epidemiol. 2014;38(3):214-226. doi:10.1016/j. canep.2014.03.012

18. Lin C, Clark R, Tu P, Bosworth HB, Zullig LL. Breast cancer oral anti-cancer medication adherence: a systematic review of psychosocial motivators and barriers. Breast Cancer Res Treat. 2017;165 (2):247-260. doi:10.1007/s10549-017-4317-2

19. Streeter SB, Schwartzberg L, Husain N, Johnsrud M. Patient and plan characteristics affecting abandonment of oral oncolytic prescriptions. Am J Manag Care. 2011;17(Suppl 5):Sp38-44.

20. Gast A, Mathes T. Medication adherence influencing factors-an (updated) overview of systematic reviews. Syst Rev. 2019;8(1):112. doi:10.1186/s13643-019-1014-8

21. Messina C, Messina M, Zanardi E. Risks and benefits from CDK inhibitors for advanced HR+ Her 2- breast cancer. Ann Oncol. 2017;28(12):3099-3100. doi:10.1093/annonc/mdx530

22. Messina C, Cattrini C, Buzzatti G, et al. CDK4/6 inhibitors in advanced hormone receptor-positive/HER2-negative breast cancer: a systematic review and meta-analysis of randomized trials. Breast Cancer Res Treat. 2018;172(1):9-21. doi:10.1007/s10549-018-4901-0

23. Li J, Huo X, Zhao F, et al. Association of cyclin-dependent kinases 4 and 6 inhibitors with survival in patients with hormone-receptor positive metastatic breast cancer: a systematic review and meta-analysis. JAMA Network Open. 2020;3(10):e2020312. doi:10. 1001/jamanetworkopen.2020.20312

24. Martin JM, Goldstein LJ. In support of CDK 4/6 inhibitors - A meta-analysis of available randomized data. JAMA Network Open. 2020;3(10):e2021062. doi:10.1001/jamanetworkopen.2020.21062
25. Baird J, Bowen K, Gleason P. Palbociclib (Ibrance ${ }^{\circledR}$ ) Utilization and Costs among 18 Million Insured Americans: managed Care Pharmacy Opportunities. presented at: AMCP: Orlando, FL, USA; 2015.

26. Guest G, Bunce A, Johnson L. How Many Interviews Are Enough? Field Methods. 2016;18(1):59-82. doi:10.1177/1525822x05279903

27. Murphy CC, Bartholomew LK, Carpentier MY, Bluethmann SM, Vernon SW. Adherence to adjuvant hormonal therapy among breast cancer survivors in clinical practice: a systematic review. Breast Cancer Res Treat. 2012;134(2):459-478. doi:10.1007/s10549-0122114-5

28. Atkinson TM, Rodríguez VM, Gordon M, et al. The Association Between Patient-Reported and Objective Oral Anticancer Medication Adherence Measures: a Systematic Review. Oncol Nurs Forum. 2016;43(5):576-582. doi:10.1188/16.Onf.576-582

29. Singhal M, Nepal B, Fisch $M$ et al. Treatment Patterns Including Adherence/Persistence With Cyclin-Dependent Kinase 4\&6 Inhibitors (CDK4\&6i) Among US Commercially Insured Women With Metastatic Breast Cancer (mBC). presented at: Virtual ISPOR Europe 2020: Milan, Italy. Value in Health, 23; 2020, Issue S2, PCN221.

30. Kimmick G, Edmond SN, Bosworth HB, et al. Medication taking behaviors among breast cancer patients on adjuvant endocrine therapy. Breast. 2015;24(5):630-636. doi:10.1016/j.breast.2015.06. 010

31. Babcock A, Ali AA, Balkrishnan R, Montero A, Diaby V. RealWorld Clinical and Economic Outcomes Associated with Palbociclib for HR-Positive/HER2 Negative Metastatic Breast Cancer: a Commentary. J Manag Care Spec Pharm. 2020;26 (7):826-831. doi:10.18553/jmcp.2020.26.7.826

32. Geynisman DM, Meeker CR, Doyle JL, et al. Provider and patient burdens of obtaining oral anticancer medications. Am J Manag Care. 2018;24(4):e128-e133.

33. Zafar SY, Peppercorn J, Asabere A, Bastian A. Transparency of Industry-Sponsored Oncology Patient Financial Assistance Programs Using a Patient-Centered Approach. J Oncol Pract. 2017;13(3):e240-e248. doi:10.1200/jop.2016.017509

34. Zullig LL, Wolf S, Vlastelica L, Shankaran V, Zafar SY. The Role of Patient Financial Assistance Programs in Reducing Costs for Cancer Patients. J Manag Care Spec Pharm. 2017;23(4):407-411. doi:10.18 553/jmcp.2017.23.4.407

35. Farano JL, Kandah HM. Targeting Financial Toxicity in Oncology Specialty Pharmacy at a Large Tertiary Academic Medical Center. J Manag Care Spec Pharm. 2019;25(7):765-769. doi:10.18553/ jmcp.2019.25.7.765

36. Olszewski AJ, Zullo AR, Nering CR, Huynh JP. Use of Charity Financial Assistance for Novel Oral Anticancer Agents. $J$ Oncol Pract. 2018;14(4):e221-e228. doi:10.1200/JOP.2017.027896
Patient Preference and Adherence

\section{Publish your work in this journal}

Patient Preference and Adherence is an international, peer-reviewed, open access journal that focusing on the growing importance of patient preference and adherence throughout the therapeutic continuum. Patient satisfaction, acceptability, quality of life, compliance, persistence and their role in developing new therapeutic modalities and compounds to optimize clinical outcomes for existing disease states are major areas of interest for the journal. This journal has been accepted for indexing on PubMed Central. The manuscript management system is completely online and includes a very quick and fair peer-review system, which is all easy to use. Visit http:// www.dovepress.com/testimonials.php to read real quotes from published authors. 